\title{
Innovative therapies in genetic diseases: Spinal muscular atrophy
}

\author{
Elena-Silvia Shelby ${ }^{1}$, Andrada Mirea ${ }^{1,2}$ \\ 1"Dr. Nicolae Robanescu" National Clinical Center for Children's Neurorehabilitation, \\ Bucharest, Romania \\ 2"Carol Davila" University of Medicine and Pharmacy, Bucharest, Romania
}

\begin{abstract}
Spinal muscular atrophy is a congenital neuromuscular disease characterized by the deterioration of the motor neurons located mainly in the anterior horns of the spinal cord, leading to progressive muscle weakness and atrophy. Globally, SMA is, after cystic fibrosis, the second cause of death due to a a genetic disease in the pediatric population. Over $95 \%$ of the total cases of SMA are represented by $5 q$ SMA, caused by biallelic mutations in the SMN1 gene (5q13.2), the rest of the SMA types being called, generically, non-5q SMA. Currently, a few genetic targeted therapies are available for $5 q$ SMA, while other innovative therapies are still in clinical trials. Early diagnosis and treatment of $5 q$ SMA have an essential role in preventing the onset and evolution of symptoms and can save the life of the patient and prevent debilitating sequelae. This article aims to briefly describe the cause and symptomatology of $5 q$ SMA as well as to make a short review of the genetic therapies available for this disease.
\end{abstract}

Keywords: spinal muscular atrophy, gene therapy, nusinersen, risdiplam, onasemnogene abeparvovec-xioi, motor neuron

\section{INTRODUCTION}

Spinal muscular atrophy (SMA), or spinal amyotrophy, is a neuromuscular genetic disease caused by the deterioration of the motor neurons located mainly in the anterior horns of the spinal cord, manifesting through progressive muscular weakness and atrophy (1). Over $95 \%$ of the total cases of SMA are caused by biallelic mutations in the SMN1 gene, located on chromosome $5 \mathrm{q}$, in the $5 \mathrm{q} 13.2$ locus, this disease being called 5q SMA, while for the rest of about 4-5\% of the SMA cases, named, generically, non-5q SMA, currently, 16 genes (such as, for example, DYNC1H1, BICD2, PLEKHG5 and ASAH1) and a still unellucidated locus are known to be involved (1-4).

Currently, 5q SMA represents, globally, the second cause of child mortality due to a genetic disease, after cystic fibrosis (5).

This article aims to briefly present the innovative and recently approved therapies for treating spinal muscular atrophy (SMA), stressing the important aspects regarding the disease and efficient therapy.

\section{SMA PHENOTYPES}

5q SMA and non-5q SMA can have very similar phenotypes, in spite of the different chromosomal location of the involved genes $(1,3)$.

With an incidence between 1 in 8,000 to one in 10,000 (1) and a global carrier frequency of 1 in 40 to 1 in 60 (3), 5q spinal muscular atrophy is characterized by progressive, bilaterally symmetric muscle weakness and atrophy (1), with a predominantly proximal onset, a more severe involvement of the lower limbs compared to the upper limbs, with the absence or marked decrease of the deep tendon reflexes and with a relative sparing of the facial, extraocular and diafragm muscles (3).

According to the clinical severity, 5q SMA can be divided into 5 types, from type 0 - the most severe to type 4 - the least severe (3).

Thus, type 0 5q SMA, the rarest type of 5q SMA (less than $1 \%$ of the total cases) has a prenatal onset characterized by the absence of fetal movements. Children with this disease never acquire sitting or 
head control and present marked hypotonia and severe muscle weakness, facial diplegia, contractures, absent response to stimuli, dysphagia and breathing insufficiency. A large number of these cases also present congenital cardiac defects. Death occurs before the age of 6 months, sometimes even after a few weeks of life $(5,6)$.

Type $15 \mathrm{q}$ SMA (Werdnig-Hoffmann disease or SMA with infantile onset) represents about $45 \%$ of the total number of cases and occurs before the age of 6 months. The patients are very hypotonic, with predominantly proximal muscle weakness, weak cry, breathing insufficiency or abdominal breathing, difficulties in suckling, swallowing difficulties, tongue fasciculation and a high risk of aspiration pneumonia; these patients never acquire sitting; in the absence of assisted ventilation, death occurs in the first two years of life by breathing insufficiency $(3,5,6)$.

Type 2 5q SMA (Dubowitz disease) represents approximately $20 \%$ of the total number of cases. Onset is before one and a half years of age (between 6 and 18 months). Patients acquire sitting, but never achieve orthostatic position or independent ambulation $(5,6)$. Patients have predominantly proximal muscle weakness, swallowing difficulties, tongue fasciculations and breathing insufficiency (5). Usually, facial and ocular muscles are not involved (6). Life expectancy is prolonged compared to type 0 or type $15 \mathrm{q}$ SMA, as these patients can reach adulthood without treatment (3).

Type 3 5q SMA, also called Kugelberg-Welander disease or SMA with juvenile onset $(3,6)$ manifests itself after the age of 18 months and has a variable evolution. Some patients are wheelchair-bound since childhood, while others can maintain independent ambulation until adulthood. All patients acquire walking, but the predominantly proximal muscle weakness affecting mainly the lower limbs leads to frequent falls and difficulties in climbing stairs, with gradual loss of ambulation (6). Life expectancy is normal (3).

Type 4 5q SMA begins during adulthood, usually after the age of 30. Patients maintain their mobility throughout the entire lifespan. Life expectancy is normal $(3,6)$.

\section{GENETIC SUBSTRATE}

The SMN protein, which causes 5q SMA when present in an insufficient quantity (7) is ubiquitously expressed (8), predominantly in the spinal cord (2), being involved in many essential processes of cell homeostasis regulation, such as spliceosome assembly, ribonucleoprotein synthesis, messenger-RNA transport, translation, endocytosis or regulation of the ubiquitin-proteasome system. Moreover, this protein is involved in the homeostasis of cell energy, having an important role at the mitochondrial level (7).

The largest amount of SMN protein (90\%) is produced by the SMN1 gene, with a small quantity $(10 \%)$ of SMN protein produced by the SMN2 gene (2).

The SMN1 and SMN2 genes (located in the 5 q13.2 chromosomal region, with SMN1 in the telomeric region SMN2 in the centromeric region) $(2,9)$ are paralogous, the SMN2 gene being produced, throughout the evolution, through the duplication and inversion of a fragment in the $5 \mathrm{q} 13.2$ region (9). Due to their similarity and opposite direction, these genes are predisposed to frequent mutational events during crossing-over. The SMN1 and SMN2 genes are almost identical, with the exception of 7 nucleotides in intron 6, 2 nucleotides in intron 7, one nucleotide in exon 7 and one nucleotide in exon 8 (10).

The $\mathrm{C}$ to $\mathrm{T}$ transition in exon 7 of the SMN2 gene (the cytosine in this position of the SMN1 gene being replaced, in the SMN2 gene, with thymine) leads to the activation of a splicing situs, promoting the binding of several splicing-promoting proteins which remove the exon 7 of SMN2 gene. Consequently, about $90 \%$ of the SMN2 transcripts do not contain exon 7 , which leads to the formation of a truncated SMN2 protein that is quickly degraded by the ubiquitin-proteasome system (10). Therefore, because of these small structural differences between the SMN1 and SMN2 genes, in the healthy individuals, the SMN1 gene is completely functional and the splicing is done correctly, while during SMN2 splicing, exon 7 is removed in about $90 \%$ of the transcripts. Thus, in healthy individuals, only $10 \%$ of the total quantity of the SMN protein encoded by the SMN2 is functional (9).

It has been proved that the severity of the manifestations of 5q SMA is inversely proportional with the number of copies of the SMN2 gene (11).

Thus, patients with type $05 \mathrm{q}$ SMA have one copy of the SMN2 gene, patients with type 1 have two copies, patients with type 2 have three copies, patients with type 3 have three to four copies and patients with type 4 have over four copies (11) (table 1).

\section{INNOVATIVE THERAPIES IN SMA}

Currently, the most promising therapies for SMA have been proven to be either the modulation of the splicing process of SMN2 premesseger RNA or gene therapy by introducing into the nucleus, using a viral 
TABLE 1. Types of $5 q$ SMA in the pediatric practice - table made according to the expertise of „Dr. Nicolae Robănescu" National Clinical Center for Children's Neurorehabilitation, Bucharest, Romania, in accordance with the information from literature $(1,4,5,11)$

\begin{tabular}{|c|c|c|c|c|c|}
\hline SMA type & $\begin{array}{c}\text { Number of } \\
\text { SMN2 copies }\end{array}$ & $\begin{array}{c}\text { Age of first } \\
\text { symptoms }\end{array}$ & Ability to sit & $\begin{array}{c}\text { Ability to achieve } \\
\text { orthostatic position }\end{array}$ & Ability to walk \\
\hline 0 & $0-1$ & In utero & No & No & No \\
\hline 1 & 2 & $<6$ months & No & No & No \\
\hline 2 & $2-3$ & $6-18$ months & Yes & Yes & Yes, assisted (with support) \\
\hline 3 & $3-4$ & $>18$ months & Yes & Yes & Yes \\
\hline
\end{tabular}

vector, a healthy SMN1 gene meant to replace the mutant one (12).

Nusinersen (known under the commercial name of Spinraza) was approved in the United States of America in December of 2016 (13) and in Europe in May of 2017 (14) and consists of antisense modified oligonucleotides which have the capacity of binding to ISS-N1 (intronic splice silencer N1) in intron 7 of the SMN2 pre-messenger RNA. This leads to the synthesis of a complete, functional SMN protein, which will not be degraded (15).

The drug has resulted in statistically significant improvements of the motor function and survival in all types of 5q SMA (16).

The improvement of the motor status was all the more increased as the therapy was started earlier and the manifestations of the disease were more severe (17). The therapy has made acquiring and keeping the motor milestones possible, while improving the respiratory function and nutritional status and decreasing the number of hospital admissions, at the same time (17).

The drug has no important side effects (17), among the potential adverse reactions being headaches, vomiting, fever, constipation, infections of the lower respiratory tract as well as lumbar pain (18).

Administration is done intrathecally, according to an injection calendar, the first 3 doses being administered at an interval of 14 days, the fourth, 30 days from the third dose, after which a maintainance dose is administered every 4 months, for the rest of the patient's life $(18,19)$.

In Romania, the cost of nusinersen is covered by the National Health Insurance House through the National Rare Disease Program (20).

Nusinersen was first administered in Romania in September, 2018 in the „Dr. Nicolae Robănescu”, National Clinical Center for Children's Neurorehabilitation in Bucharest. Gradually, other centers from the country started to offer this therapy for 5q SMA patients $(20,21)$. Our experience in administering nusinersen has shown that this therapy is the more efficient, the sooner it is started. Following the initiation of this therapy our patients have shown a signifi- cant improvement of motor function and a marked increase in the quality of life. Moreover, the necessity of costly assisting devices, which represent a supplementary financial burden for the parents and for the health system, was reduced. Thus, among the patients which received nusinersen shortly after the onset of the disease, we mention: a type I SMA patient who has achieved walking with support at the age of three, a type II SMA patient who has achieved independent ambulation when he was one year and 11 months old, as well as a patient with two SMN2 copies (who has a brother with type II SMA) who achieved autonomous ambulation and climbing the stairs when she turned one. The latter patient had normal motor development.

Onasemnogene abeparvovec-xioi (known under the commercial name of Zolgensma) is a gene therapy recommended for the treatment of patients under two years of age which have biallelic mutations in the SMN1 gene, in the USA, and for the treatment of patients with biallelic mutations in SMN 1, as well as clinical diagnosis of type 1 SMA and up to three copies of the SMN2 gene, in Europe (22). The therapy consists in introducing the SMN1 transgene into the cell's nucleus using, as a vector, AAV-9 (adeno-associated virus 9). Administration is made through a single i.v. perfusion (23).

The effects of the treatment have proven to be significant: following therapy, the normal levels of SMN protein were restored in the motor neurons, the motor function was shown to be significantly increased and the life expectancy of patients with SMA was significantly improved, with effects persisting 6 years after the first administration (23). The most frequent side effects of onasemnogene abeparvovec-xioi were increased liver enzymes and vomiting, therefore the level of transaminases must be monitored for at least 3 months following administration of this therapy (23). Furthermore, in March of 2021 Novartis warned on the risk of thrombotic microangiopathy (thrombocytopenia, hemolitic anemia and renal failure, with varied manifestations - bruising, convulsions, oliguria) following the administration of onasemnogene abeparvovec-xioi and has recommended, in this re- 
gard, careful monitorization of the hemoleucogram and creatininemia. Up to March of 2021, the therapy had been administered to 800 patients among which 5 , with ages between 4 and 23 months, had been confirmed with thrombotic microangiopathy (24).

Onasemnogene abeparvovec-xioi was approved by FDA on the 24th of May, 2019, for 5q SMA type 1, 2 and 3 patients (25). On the 19th of May, 2020, the Swiss pharmaceutic company Novartis obtained conditional approval (the benefits of the immediate availability overcome the risks, but the safety of this medicine has not been studied enough, yet) of the European Comission for the treatment of 5q SMA patients with biallelic mutations in the SMN1 gene and up to 3 copies of the SMN2 gene, with a weight of up to 21 kilograms $(26,27)$.

In December 2019, Avexis, a subsidiary of Novartis, announced the launch of a global acces program (global MAP - global managed-access programme) for Zolgensma $(28,29)$. The program was launched in January of 2020 and aims to distribute doses of this drug in countries throughout the world where its use has not been approved yet, with the possibiliy of prolongation, after a reevaluation, and of introducing new doses every 6 months (29). The program is addressed to children with ages under 2 and genetically confirmed 5q SMA, regardless of the type of SMA, age of onset or previous treatments (28). The attending physicians must send a request for treatment of their patients and, once patient eligibility is established, an authorisation from the patient's country must be obtained (28). Starting with February of 2020, lotteries take place twice a week, and the patients which have not been selected for treatment are automatically signed in for the next lottery (28). The requests for patients in countries where the therapy with Onasemnogene abeparvovec-xioi has already been approved are automatically rejected (28).

Thus, in July of 2020, the first gene therapy ever performed in Romania was done in the „Dr. Nicolae Robănescu", National Clinical Center for Children's Neurorehabilitation, following the administration to a patient with SMA, within the MAP program (30).

Up to now, we don't have any evidence that this therapy has brought additional benefits to our patients, as they had also been concomitantly receiving nusinersen, a therapy which has been constantly improving their motor function. Nevertheless, we remarked a substantial improvement in the deglutition function of a 12 months old male patient. About three months since gene therapy was performed, the patient didn't need the nasogastric tube anymore and feeding could be done orally. Furthermore, it's im- portant to say that a one year and seven months old female patient with type I SMA presented, in the first week following gene therapy, multiple organ failure requiring peritoneal dialysis for about one month and intubation and mechanical ventilation throughout the entire hospitalization period, up to her death, which occured about 2,5 months after gene therapy.

The rest of the four patients receiving gene therapy presented increases of the transaminase levels (three times the maximum normal values), which required cortisone administration up to 5 months after dosing. Only one patient had demineralization fractures (Milchmann Looser), possibly as a consequence of the prolonged administration of prednisone.

Currently, Novartis has renewed this program with another 100 doses planned for 2021 (31).

After the FDA's approval and EMA's conditional authorization for the administration of onasemnogene-aboparvovec-xioi were obtained, there were a few cases of pediatric patients that followed both types of treatment, concurrently. As it is already known, the two types of therapy have different mechanisms of action and the long-term effects of the concurrent administration of these drugs are not known yet. According to a study from 2020, made on only 5 patients, the nusinersen-onasemnogene-abeparvovec-xioi combination was well tolerated (32). According to our information obtained after consulting various international SMA experts, concurrent administration of the two drugs cannot bring any benefit.

Regarding nusinersen's efficiency compared to onasemnogene abeparvovec, a study (33) from 2019 made, indirectly, on children up to 2 years of age with type 1 SMA which has compared two clinical trials for onasemnogene abeparvovec (AVXS-101CL-101) and nusinersen (ENDEAR), analysing the global survival rate, survival with no events and motor improvement rate, has concluded that onasemnogene abeparvovec seems to be more efficient in regards to the global survival rate, motor function and need for assisted ventilation, compared to nusinersen.

Risdiplam (RG7916, RO7034067) is an oral therapy developed by Roche and Genetech in collaboration with PTC Therapeutics and SMA Foundation (34), which acts on the splicing process of SMN2 premessenger RNA, promoting the inclusion of exon 7 with the synthesis of complete SMN2 transcripts which lead to the encoding of a functional SMN protein (35). The mechanism of action is similar to nursinersen (36). Risdiplam binds the two SMN2 premessenger RNA sites, namely the 5' splicing site in intron 7 (5'ss) and the exonic splicing activator 2 (ESE2) from exon 7, with the subsequent recruitment of U1s- 
nRNP, a component of the spliceosome which promotes the inclusion of exon 7 (37). Risdiplam's oral administration route makes this drug a more attractive option than nusinersen in regards to bioavailability (36). The administration of risdiplam for 12 months has shown a double increase in the bodily level of SMN protein and a good distribution of this drug in the central nervous system and peripheric nervous system of patients with SMA (36). The SUNFISH study proved that Risdiplam significantly improved the motor function of type 2 and 3 SMA patients (34). Furthermore, the FIREFISH study showed that this drug is very efficient and safe for treating type 1 SMA patients (38). The most common side effects are fever, diarrhea, upper respiratory tract infection, otitis, pneumonia, constipation, vomiting, cough and inflammation of the upper respiratory tract, in the above-mentioned order (39).

In November of 2019, the FDA has granted priority to this drug, as a NDA (New Drug Application) (40). In January of 2020, the pharmaceutical company Roche made this therapy available globally through a compassionate program (Pre-Approval Access/Compassionate Use Programme) (35). This program made the administration of risdiplam possible in April of 2020, in the „Dr. Nicolae Robănescu” National Clinical Center for Children's Neurorehabilitation (30).

We could thus notice that this therapy is the more efficient, the sooner it is started. Moreover, we have noticed an improvement in the ventilation function of the tracheostomized and permanently ventilated patients, through the increase of the respiratory trigger with the doubling of the number of respirations initiated by the patient. Except for one type I SMA patient, aged 7, who gained 800 grams following the first month of treatment, no other side effects were noted in the other 9 patients treated during this compassional use program. With the exception of the two patients needing permanent ventilation, who didn't have any active movements before the therapy was initiated, all patients had a significant increase in their motor function. Thus, we especially noted the evolution of a type II SMA patient who can currently walk and climb stairs with support, without alternating the lower limbs. We believe her outstanding progress is due to rapid initiation of the therapy.

Risdiplam received the FDA's approval in August of 2020 for the treatment of children with SMA over 2 months of age, under the commercial name of Evrysdi (41). In March of 2021, after obtaining EMA's approval, risdiplam obtained from the European Commision the commercial authorization for Europe (42).

Recent data indicates that risdiplam could cause infertility in males, this subject is still being currently studied $(43,44)$.

\section{CONCLUSIONS}

The availability of targeted therapies acting on SMN2's premessenger RNA, as well as of gene therapy meant to replace the mutant SMN1 gene with a healthy one, have dramatically changed the evolution of the clinical picture of SMA, creating new phenotypes. Thus, type I SMA patients who, according to the definition of the disease could never acquire sitting, can now even walk with support, and type II SMA patients can acquire autonomous ambulation. Given the fact that the treatment is more efficient the sooner it is started, early administration being able to stop the development of irreversible neurological sequelae, rapid recognition of the symptoms of this disease and redirection of the patient to a pediatric neurologist and a center which performs targeted genetic therapy is essential. The existence of these therapies, among which one is reimbursed by the National Health Insurance House, together with the invaluable efficiency of early treatment (before onset of symptoms), requires an important effort with invaluable benefits for the patients: starting newborn screening for SMA in Romania.

Conflict of interest: none declared Financial support: none declared

\section{REFERENCES}

1. Spinal muscular atrophy [Internet]. [cited 2021 May 17]. Available at: https://ghr.nlm.nih.gov/condition/spinal-muscular-atrophy\#statistics.

2. SMN1 gene survival of motor neuron 1, telomeric [Internet]. [cited 2021 May 17]. Available at: https://medlineplus.gov/genetics/gene/ smn1/\#location.

3. Farrar MA, Kiernan MC. The genetics of spinal muscular atrophy: progress and challenges. Neurotherapeutics. 2015;12(2):290-302.

4. Peeters K, Chamova T, Jordanova A. Clinical and genetic diversity of SMN1-negative proximal spinal muscular atrophies. Brain. 2014;137(11):2879-96

5. Keinath MC, Prior DE, Prior TW. Spinal Muscular Atrophy: Mutations, Testing, and Clinical Relevance. Appl Clin Genet. 2021;14:11.

6. Muscular Distrophy Association [Internet]. Available at: https://www. mda.org/disease/spinal-muscular-atrophy/types.

7. Chaytow H, Huang Y-T, Gillingwater TH, Faller KME. The role of survival motor neuron protein (SMN) in protein homeostasis. Cell Mol Life Sci. 2018;75(21):3877-94.

8. Monani UR. Spinal muscular atrophy: a deficiency in a ubiquitous protein; a motor neuron-specific disease. Neuron. 2005;48(6):885-95.

9. Kirwin SM, Vinette KMB, Gonzalez IL, Abdulwahed H Al, Al-Sannaa $\mathrm{N}$, Funanage VL. A homozygous double mutation in SMN 1: A 
complicated genetic diagnosis of SMA. Mol Genet genomic Med. 2013;1(2):113-7.

10. Hua Y, Vickers TA, Baker BF, Bennett CF, Krainer AR. Enhancement of SMN2 exon 7 inclusion by antisense oligonucleotides targeting the exon. PLoS Biol. 2007;5(4):e73.

11. Butchbach MER. Copy number variations in the survival motor neuron genes: implications for spinal muscular atrophy and other neurodegenerative diseases. Front Mol Biosci. 2016;3:7.

12. Chen $\mathrm{TH}$, Wang $\mathrm{CH}$. Emerging Innovative Therapies of Spinal Muscular Atrophy: Current Knowledge and Perspectives. Front Clin Drug Res Neurol Disord. 2020;8:1.

13. AHFS approved Spinraza [Internet]. Available at: https://www. ahfsdruginformation.com/spinraza-nusinersen-approved/.

14. EMA Nusinersen approved [Internet]. Available at: https://www.ema. europa.eu/en/medicines/human/orphan-designations/eu312976.

15. Summarry of Product INN Nusinersen [Internet]. Available t: https:// www.ema.europa.eu/en/documents/product-information/spinrazaepar-product-information_en.pdf.

16. SMA News Today - Spinraza [Internet]. Available at: https:// smanewstoday.com/spinraza-nusinersen/.

17. Figueiredo M. Motor Function Improved after Spinraza [Internet]. Available at: https://smanewstoday.com/2020/06/24/spinrazaimproves-motor-function-after-1-year-children-sma-type-1-2-real-lifestudy-francel.

18. Intrathecal administration with SPINRAZA® (nusinersen) [Internet]. Available at: https://www.spinraza.com/content/dam/commercial/ spinraza/caregiver/en_us/pdf/SPZ-US-0603-Intrathecal-DiscussionTool.pdf.

19. Healthcare Information Safety - Spinraza [Internet]. Available from: https://www.spinraza.com/en_us/home/taking-spinraza/dosing.html. at.

20. National Health Insurance House. Press release - Romania has started treating patients with spinal amyotrophy with the drug nusinersen. [Online]. [Internet]. Available at: http://www.cnas.ro/post/ type/local/comunicat-romania-a-inceput-tratarea-pacientilor-cuamiotrofie-spinala-cu-medicamentul-nusinersen.html.

21. 360medical [Internet]. Available at: https://360medical.ro/stiri/ pacientii-adulti-cu-atrofie-musculara-spinala-inclusi-in-tratament-decnas-dupa-protestele-din-ultimele-luni/2019/12/23/..

22. Zolgensma FDA [Internet]. Available at: https://www.fda.gov/ vaccines-blood-biologics/zolgensma.

23. Mahajan R. Onasemnogene abeparvovec for spinal muscular atrophy: the costlier drug ever. Int J Appl Basic Med Res. 2019;9(3):127.

24. EMA thrombotyc microangiopathy [Internet]. Available at: https:// www.ema.europa.eu/en/documents/dhpc/direct-healthcareprofessional-communication-dhpc-zolgensma-onasemnogeneabeparvovec-risk-thrombotic_en.pdf.

25. FDA. Statement on data accuracy issues with recently approved gene therapy. [2019] [Internet]. Available at: https://www.fda.gov/ news-events/press-announcements/statement-data-accuracy-issuesrecently-approved-gene-therapy.

26. AVEXIS EC APPROVAL - Day 1 [Internet]. Available at: https://www. novartis.com/news/media-releases/avexis-receives-ec-approval-and- activates-\%22day-one\%22-access-program-zolgensma-only-genetherapy-spinal-muscular-atrophy-sma.

27. Biopharmadive [Internet]. Available at: https://www.biopharmadive. com/news/novartis-zolgensma-europe-approval/578320/

28. controversial programme [Internet]. Available from: https://www. sma-europe.eu/news/avxs-101-zolgensma-to-be-made-availableglobally-through-a-controversial-programme/.

29. global access zolgensma [Internet]. Available at: https:// smanewstoday.com/2019/12/28/novartis-global-access-program-forzolgensmal

30. No Title [Internet]. Available at: http://www.recuperarecopii.ro/assets/ images/comunicate/new doc 2020-07-22 12.41.05.pdf.

31. Company News HQ. Novartis. Novartis Gene Therapies Recommits to Global Man-aged Access Program for 2021. [Online] [Internet]. Available at: https://www.companynewshq.com/company-news/ pharmaceutical-company-news/novartis-gene-therapies-recommitsto-global-managed-access-program-for-2021/.

32. Harada Y, Rao VK, Arya K, Kuntz NL, DiDonato CJ, NapchanPomerantz G, et al. Combination molecular therapies for type 1 spinal muscular atrophy. Muscle Nerve. 2020;62(4):550-4.

33. Dabbous O, Maru B, Jansen JP, Lorenzi M, Cloutier M, Guérin A, et al. Survival, motor function, and motor milestones: comparison of AVXS-101 relative to nusinersen for the treatment of infants with spinal muscular atrophy type 1. Adv Ther. 2019;36(5):1164-76.

34. Risdiplam Leads to Motor Skills Gains or Stability in SMA Types 2 and 3, SUNFISH Trial reports. [Online]. 2020 [Internet]. Available at: https://smanewstoday.com/2020/02/06/risdiplam-leads-to-motorskills-gains-or-stability-in-sma-type-2-and-3-sunfish-trial-reports/.

35. Compassioante use Risdiplam [Internet]. Available at: https://www. treatsma.uk/treatments/risdiplam/.

36. Drug Bank Risdiplam [Internet]. Available at: https://www.drugbank. ca/drugs/DB15305.

37. Sivaramakrishnan M, McCarthy KD, Campagne S, Huber S, Meier S, Augustin A, et al. Binding to SMN2 pre-mRNA-protein complex elicits specificity for small molecule splicing modifiers. Nat Commun. 2017;8(1):1-13.

38. FIREFISH Risdiplam [Internet]. Available at: https://www.treatsma. uk/2020/01/risdiplam-is-effective-in-treating-babies-with-sma-type-1/.

39. Roche announce preliminary results [Internet]. Available at: https:// www.roche.com/media/releases/med-cor-2018-10-03.htm.

40. No Title [Internet]. Available at: https://smauk.org.uk/risdiplam.

41. Dhillon S. Risdiplam: first approval. Drugs. 2020;1-6.

42. EMA Evrysdi approved [Internet]. Available at: https://www. europeanpharmaceuticalreview.com/news/149341/evrysdi-approvedfor-treatment-of-spinal-muscular-atrophy-in-europe/.

43. Ravi B, Chan-Cortés MH, Sumner CJ. Gene-targeting therapeutics for neurological disease: lessons learned from spinal muscular atrophy. Annu Rev Med. 2021;72:1-14.

44. Mathews KD, lannaccone ST. Combination molecular therapies for spinal muscular atrophy: How much is enough? Wiley Online Library; 2020. 